\title{
Hypernetted-chain study of broken rotational symmetry states for the $\nu=1 / 3$ fractional quantum Hall effect and other fractionally filled Landau levels
}

\author{
Orion Ciftja and C. Wexler \\ Department of Physics and Astronomy, University of Missouri-Columbia, Columbia, Missouri 65211
}

(November 6, 2018)

\begin{abstract}
We investigate broken rotational symmetry (BRS) states for the fractional quantum Hall effect (FQHE) at 1/3-filling of the valence Landau level (LL). Recent Monte Carlo calculations by Musaelian and Joynt [J. Phys.: Condens. Matter 8, L105 (1996)] suggest that Laughlin's state becomes unstable to a BRS state for some critical finite thickness value. We study in detail the properties of such state by performing a hypernetted-chain calculation that gives results in the thermodynamic limit, complementing other methods which are limited to a finite number of particles. Our results indicate that while Laughlin's state is stable in the lowest LL, in higher LLs a BRS instability occurs, perhaps indicating the absence of FQHE at partial fillings of higher LLs. Possible connections to the newly discovered liquid crystalline phases in higher LLs are also discussed.
\end{abstract}

PACS: 73.43.-f, 73.43.Lp, 73.43.Nq, 73.20.Mf, 64.70.Md.

\section{INTRODUCTION}

Recently, a plæthora of new phenomena has emerged in the transitional regions between different plateaus of the Hall conductancel 4 of Landau levels (LL) with in$\operatorname{dex} L \geq 2$. Near half-filling of the valence LL, extreme anisotropy has been measured in the magnetotransport below temperatures ca. $100 \mathrm{mK}$, 13 accompanied by smooth non-linearities. In addition, reentrant integer quantum Hall effect (RIQHE) regions with striking breakdown features and new phase transitions (presumed to be quantum in rigin 5 ) have been seen near $1 / 4$ filling of the valence LL.6

The anisotropic behavior has been attributed to the formation of a nematic phase of the two dimensional electron system (2DES) which undergon nematic to isotropic transition at higher temperatures.5 Similarly, the reentrant regions are believed to be "bubble phases" similar to Wigner crystals, but with several electrons per bubble, or possibly new electronic hexatic states.5

The motivation of our work is to study these numerous liquid crystalline phases present in partially filled LLs by means of many-body trial wavefunctions with broken rotation symmetry (BRS), similar to those proposed by Musaelian and Joynt (MJ) 8 in the context of the fractional quantum Hall effect (FQHE) (which are essentially generalizations of Laughlin's wavefunction 9 ):

$$
\begin{aligned}
& \Psi_{\alpha}\left(z_{1}, \ldots, z_{N}\right)= \\
& \prod_{i>j}^{N}\left(z_{i}-z_{j}\right)\left(z_{i}-z_{j}-\alpha\right)\left(z_{i}-z_{j}+\alpha\right) e^{-\frac{1}{4} \sum_{i=i}^{N}\left|z_{i}\right|^{2}}
\end{aligned}
$$

where $z_{j}=x_{j}+i y_{j}$ is the complex $2 \mathrm{D}$ coordinate of $j$-th electron and $\alpha$ is a complex number (we work in units of the magnetic length: $l_{0}^{2}=\hbar / e B=1$ ). This wavefunction represents a homogeneous liquid state with filling factor $\nu=1 / 3$, lies entirely in the lowest LL (LLL), and for $\alpha \neq 0$ has nematic order (for $\alpha=0$ we recover Laughlin's wavefunction, which is obviously isotropic).
This wavefunction represents, therefore, a good starting point to consider nematic QH systems (even though the filling factor is "incorrect" for the newly discovered anisotropic states, see below for further analysis), by facilitating the systematic study the energy dependence of BRS states for diverse physical parameters (LL index, width of the 2DES, etc). In addition, the possibility of BRS states for $\nu=1 / 3$ is intriguing by itself since for $\alpha \neq 0$ highly damped low-energy modes exist, 10 strongly modifying the dynamics and possibly suppressing the FQHE.

In Ref. 8 MJ investigated the possibility of BRS in a FQHE system using Eq. (11) and performing Monte Carlo (MC) simulations in a disc geometry. Their results suggest that the Laughlin fluid becomes unstable towards BRS states in quantum wells whose thickness exceeds a critical value depending on the electronic density.

In this work we study the BRS state for $1 / 3$-filling of the valence $\mathrm{LL}$ (i.e. $\nu=M+1 / 3$ with $M$ integer by using the hypernetted-chain (HNC) method 11, 13,16 This method allows us to compute physical quantities in the thermodynamic limit, without the limitations of using a finite number of particles that hinder other techniques, where the extrapolation to the thermodynamic limit is not totally unambiguous. We find that, contrary to MJ's results, the Laughlin state is stable in the LLL, whereas a BRS instability is possible in higher LLs (perhaps indicating, analogously to the arguments of Ref. 17, why there is no ordinary FQHE at, e.g. $\nu=7 / 3$ ).

In Sec. II we present the basic theoretical calculations needed to determine the stability of an isotropic or BRS state. A detailed description of the HNC formalism in the context of the BRS wave function is given in Sec. III. The results for the BRS state in the LLL and their extension in higher Landau levels are discussed in Sec. IV. Finally, in Sec. V we discuss our results, and analyze how they can be extended to more realistic filling factors. 


\section{BASIC THEORY}

In this work we propose to study the stability of different states by using trial wavefunctions like Eq. (11). We are interested, therefore, to calculate the energy in each of these states to find the optimum value for the sole free parameter $\alpha$.

We first consider the situation in the LLL, namely the state with $\nu=1 / 3$. Since the BRS wave function is completely in the LLL the kinetic energy per particle is quenched at the lowest cyclotron energy

$$
\frac{1}{N} \frac{\left\langle\Psi_{\alpha}|\hat{K}| \Psi_{\alpha}\right\rangle}{\left\langle\Psi_{\alpha} \mid \Psi_{\alpha}\right\rangle}=\frac{1}{2} \hbar \omega_{c}
$$

where $\omega_{c}=e B / m$ is the cyclotron frequency. The potential, or correlation, energy per electron is:

$$
E_{\alpha}=\frac{1}{N} \frac{\left\langle\Psi_{\alpha}|\hat{V}| \Psi_{\alpha}\right\rangle}{\left\langle\Psi_{\alpha} \mid \Psi_{\alpha}\right\rangle}=\frac{\rho}{2} \int d^{2} r V(r)[g(\mathbf{r})-1],
$$

where $V$ represents the electron-electron, electronbackground, and background-background interaction; and $g(\mathbf{r})$ is the (angle-dependent) pair distribution function given by

$$
g(\mathbf{r})=\frac{N(N-1)}{\rho^{2}} \frac{\int d^{2} r_{3} \cdots d^{2} r_{N}\left|\Psi_{\alpha}\left(\mathbf{r}_{1} \cdots \mathbf{r}_{N}\right)\right|^{2}}{\int d^{2} r_{1} \cdots d^{2} r_{N}\left|\Psi_{\alpha}\left(\mathbf{r}_{1} \cdots \mathbf{r}_{N}\right)\right|^{2}}
$$

where $\mathbf{r}=\mathbf{r}_{2}-\mathbf{r}_{1}$. The following sum rule can be easilty proven $\rho \int d^{2} r[g(\mathbf{r})-1]=-1$, and is a convenient check for numerical procedures. For an ideal $2 \mathrm{D}$ sample the interaction is a pure Coulomb potential $V(r) \simeq e^{2} / \epsilon r$, while in samples with finite thickness a reasonable choice is the Zhang Das Sarma (ZDS) potential $18 \mathrm{~V}(r)=e^{2} / \epsilon \sqrt{r^{2}+\lambda^{2}}$, where $\lambda$ is of the order of the sample thickness. Alternatively, the correlation energy can be computed in reciprocal space:

$$
E_{\alpha}=\frac{1}{2} \int \frac{d^{2} q}{(2 \pi)^{2}} \tilde{V}(q)[S(\mathbf{q})-1]
$$

where $\tilde{V}(q)$ is the 2D Fourier transform $1 \mathrm{~g}(\mathrm{FT})$ of $V(r)$ and $S(\mathbf{q})$ is the static structure factor:

$$
S(\mathbf{q})-1=\rho \mathrm{FT}[g(r)-1] .
$$

While both $g(\mathbf{r})$ and $S(\mathbf{q})$ are angle-dependent (e.g. see Figs. 11 and 2), because the interaction potential is centrally symmetric, the energy $E_{\alpha}$ depends only on the angle-averaged pair distribution function or static structure factor defined as:

$$
\bar{g}(r)=\int_{0}^{2 \pi} \frac{d \theta}{2 \pi} g(\mathbf{r}), \quad \bar{S}(q)=\int_{0}^{2 \pi} \frac{d \theta_{q}}{2 \pi} S(\mathbf{q}) .
$$

The determination of either the pair distribution function or the structure factor is generally a complicated integral problem that needs to be solved for each LL. However, its is known that if transitions to other LLs are neglected (i.e. a single- $L L$ approximation), $g(\mathbf{r})$ and $S(\mathbf{q})$ at higher LL are simply related to those at the LLL $(L=0)$ by means of a convolution or product respectively. We will apply this approximation (which, moreover, quenches the kinetic energy in higher LLs as well). It is then, sufficient to compute these distribution functions once in the LLL and then the correlation energy per electron is given by

$$
E_{\alpha}^{L}=\frac{1}{2} \int \frac{d^{2} q}{(2 \pi)^{2}} \tilde{V}_{\mathrm{eff}}(q)[S(\mathbf{q})-1],
$$

where $\tilde{V}_{\text {eff }}(q) \equiv \tilde{V}(q)\left[L_{L}\left(q^{2} / 2\right)\right]^{2} . \quad L_{L}(z)$ are Laguerre polynomials, and $S(\mathbf{q})$ is calculated in the LLL $(L=0)$.

In what follows we compute $g(\mathbf{r})$ and $S(\mathbf{q})$ in the LLL using the HNC method.

\section{THE HNC METHOD FOR THE BROKEN ROTATIONAL SYMMETRY STATE}

Integral equation techniques such as the HNC theory 11.13 allow an accurate evaluation of the pair distribution function and related quantities associated with a Jastrow wave function. In particular they are extremely useful for calculations that are performed in the thermodynamic limit. They have been videly used in the study of classical14 and quantum fluids 16,15 However the HNC method for the BRS wave function is a slightly different from that for the Laughlin wave function, since correlations and related quantities depend on both distance and relative angle between a pair of particles.

The main quantity to be calculated in a HNC expansion is the pair distribution function $g(\mathbf{r})$ [Eq. (4)], or equivalently the structure factor $S(\mathbf{q})$ [Eq. (6)]. These may then be used in conjunction with Eqs. (3), (5), or (8) to determine the energy per electron for arbitrary values of the BRS parameter $\alpha$ or the $2 \mathrm{D}$ width $\lambda$.

Although the BRS wave function is a Fermi wave function, its modulus square,

$$
\left|\Psi_{\alpha}\left(z_{1}, \ldots, z_{N}\right)\right|^{2}=e^{\sum_{i>j}^{N} u\left(z_{i}-z_{j}\right)} e^{-\sum_{i=1}^{N} \frac{\left|z_{i}\right|^{2}}{2}},
$$

where $u(z)=\ln |z|^{2}+\ln |z-\alpha|^{2}+\ln |z+\alpha|^{2}$, can be viewed as a symmetric Jastrow wave function with pair correlations and single-particle terms. Therefore it is possible to apply the Bose HNC formalism.20 In order to compute Eq. (4) one needs some small parameter in which to expand perturbatively (and re-sum a subset of diagrams). For standard systems like Bose liquid ${ }^{4} \mathrm{He}$, the pair correlation is short-range and heals to 1 for large distances, therefore the function $\exp \left[u\left(r_{i j}\right)\right]-1$ provides a possible expansion parameter [note that in order to apply the Bose HNC expansion, the correlation (pseudo) potential has to satisfy the conditions: $u\left(r_{i j} \rightarrow 0\right) \rightarrow-\infty$ and $\left.u\left(r_{i j} \rightarrow+\infty\right) \rightarrow 0\right]$. In the case of the BRS wave function, the correlation (pseudo) potential is logarithmically long-range, however it is possible to extend the method formally by splitting all quantities to compute 
into a short- and long-range parts (see below). It can be shown that the pair distribution function can be expressed as a series of cluster terms associated with linked diagrams and will be given from the following HNC equations:

$$
\begin{aligned}
& X\left(\mathbf{r}_{12}\right)=e^{u\left(\mathbf{r}_{12}\right)+N\left(\mathbf{r}_{12}\right)+E\left(\mathbf{r}_{12}\right)}-N\left(\mathbf{r}_{12}\right)-1 \\
& N\left(\mathbf{r}_{12}\right)=\rho \int d^{2} r_{3} X\left(\mathbf{r}_{13}\right) \cdot\left[X\left(\mathbf{r}_{32}\right)+N\left(\mathbf{r}_{32}\right)\right] \\
& g\left(\mathbf{r}_{12}\right)=1+X\left(\mathbf{r}_{12}\right)+N\left(\mathbf{r}_{12}\right)
\end{aligned}
$$

The quantities $X\left(\mathbf{r}_{12}\right)$ and $N\left(\mathbf{r}_{12}\right)$ represent the sum of the so-called composite and nodal diagrams respectively and $E\left(\mathbf{r}_{12}\right)$ is the sum of elementary diagrams. The generation of diagrams contributing to $g\left(\mathbf{r}_{12}\right)$ must go through a self-consistent procedure. As a first approximation (and a good one) we take the $\mathrm{HNC} / 0$ approximation where the " 0 " denotes the neglect of elementary diagrams. The summation of the nodal diagrams $N\left(\mathbf{r}_{12}\right)$ is easily performed in Fourier space.

In order to handle the 2D logarithmic (pseudo) potential $u\left(\mathbf{r}_{12}\right)$, the standard procedure is to split it into short- and long-range parts:

$$
u\left(\mathbf{r}_{12}\right)=u_{s}\left(\mathbf{r}_{12}\right)+u_{l}\left(\mathbf{r}_{12}\right),
$$

with the nodal function $N\left(\mathbf{r}_{12}\right)$ and the composite function $X\left(\mathbf{r}_{12}\right)$ similarly split:

$$
\begin{aligned}
& N\left(\mathbf{r}_{12}\right)=N_{s}\left(\mathbf{r}_{12}\right)-u_{l}\left(\mathbf{r}_{12}\right), \\
& X\left(\mathbf{r}_{12}\right)=X_{s}\left(\mathbf{r}_{12}\right)+u_{l}\left(\mathbf{r}_{12}\right) .
\end{aligned}
$$

This splitting is done subject to the following conditions:

$$
\begin{gathered}
u\left(\mathbf{r}_{12}\right)+N\left(\mathbf{r}_{12}\right)=u_{s}\left(\mathbf{r}_{12}\right)+N_{s}\left(\mathbf{r}_{12}\right), \\
N\left(\mathbf{r}_{12}\right)+X\left(\mathbf{r}_{12}\right)=N_{s}\left(\mathbf{r}_{12}\right)+X_{s}\left(\mathbf{r}_{12}\right) .
\end{gathered}
$$

Given the particular form of (pseudo) potential for the BRS wave function, we choose to decompose $u\left(\mathbf{r}_{12}\right)$ into its short-range function (going to $-\infty$ for small distances and healing to 0 for large distances) and its long-range counterpart in the following manner:

$$
\begin{aligned}
u_{s}\left(\mathbf{r}_{12}\right)= & -2 K_{0}\left(Q r_{12}\right) \\
& -2 K_{0}\left(Q\left|\mathbf{r}_{12}-\vec{\alpha}\right|\right)-2 K_{0}\left(Q\left|\mathbf{r}_{12}+\vec{\alpha}\right|\right), \\
u_{l}\left(\mathbf{r}_{12}\right)= & +2\left[\ln \left(r_{12}\right)+K_{0}\left(Q r_{12}\right)\right] \\
& +2\left[\ln \left(\left|\mathbf{r}_{12}-\vec{\alpha}\right|\right)+K_{0}\left(Q\left|\mathbf{r}_{12}-\vec{\alpha}\right|\right)\right] \\
& +2\left[\ln \left(\left|\mathbf{r}_{12}+\vec{\alpha}\right|\right)+K_{0}\left(Q\left|\mathbf{r}_{12}+\vec{\alpha}\right|\right)\right],
\end{aligned}
$$

where $K_{0}(x)$ is the modified Bessel function, and $Q$ is cut-off parameter of order 1 . We recall that the 2D FT 19 of $u_{l}\left(\mathbf{r}_{12}\right)$ is:

$$
\tilde{u}_{l}(\mathbf{q})=-\frac{4 \pi Q^{2}}{q^{2}\left(q^{2}+Q^{2}\right)}\left(1+e^{i \mathbf{q} \cdot \vec{\alpha}}+e^{-i \mathbf{q} \cdot \vec{\alpha}}\right) .
$$

The final set of equations is solved by initially setting $N_{s}\left(\mathbf{r}_{12}\right)=0$ in Eq. (10), then obtaining $X_{s}(\mathbf{q})=$
$\mathrm{FT}\left[X_{s}\left(\mathbf{r}_{12}\right)\right]$ which can be used to compute $\tilde{X}(\mathbf{q})=$ $\tilde{X}_{s}(\mathbf{q})+\tilde{u}_{l}(\mathbf{q})$. Using the convolution theorem we find $\tilde{N}(\mathbf{q})=\rho \tilde{X}(\mathbf{q})^{2} /[1-\rho \tilde{X}(\mathbf{q})]$ and easily obtain $\tilde{N}_{s}(\mathbf{q})=$ $\tilde{N}(\mathbf{q})+\tilde{u}_{l}(\mathbf{q})$. The last step is to perform an inverse $2 \mathrm{D}$ FT on $\tilde{N}_{s}(\mathbf{q})$ to obtain the new $N_{s}\left(\mathbf{r}_{12}\right)$. This procedure is repeated until a desired accuracy is reached. After convergence the pair distribution function is given by

$$
g\left(\mathbf{r}_{12}\right)=1+X_{s}\left(\mathbf{r}_{12}\right)+N_{s}\left(\mathbf{r}_{12}\right) .
$$

Simultaneously, the static structure factor is given by

$$
S(\mathbf{q})=1+\rho\left[\tilde{X}_{s}(\mathbf{q})+\tilde{N}_{s}(\mathbf{q})\right] .
$$

The computation of such functions allows us to find the interaction energy and other related quantities.

\section{RESULTS AND DISCUSSIONS}

In the present work we applied the HNC theory to study the BRS state at filling $1 / 3$ of an arbitrary LL (in the single-LL approximation). For the sake of simplicity we neglected the elementary diagrams (i.e. the so-called HNC/0 approximation). This allows us to determine to a reasonable accuracy the pair distribution function and the static structure factor. In order to compare the $\alpha=0$ (Laughlin) state with the $\alpha \neq 0$ (BRS) state we studied the properties of the BRS wave function for several $\alpha$-s with magnitudes between 0 to 3 (in general $\alpha=|\alpha| e^{i \theta_{\alpha}}$, without loosing generality we considered only $\theta_{\alpha}=0$ ).

\section{A. Pair distribution function and structure factor}

In Fig. 1 we plot the pair distribution function $g(\mathbf{r})$ for $\alpha=2$ (top, center panels), and the angle-averaged pair distribution function $\bar{g}(r)$ corresponding to $\alpha=0$, 1, 2 and 3 (bottom panel). It is interesting to note the splitting of the triple node at the origin, the noticeable angle-dependence of $g(\mathbf{r})$, and the change in the small- $r$ behavior of $\bar{g}(r)$ which switches from $\propto r^{6}$ (for $\alpha=0$ ) to $\propto r^{2}$ as $\alpha$ is increased. In addition, we mention the following generic properties more or less valid for any of the $\alpha$-s we have considered:

1. For $\alpha \neq 0$ there is at least one pair distribution function that has an additional zero (besides the zero at $r=0$ ) at inter-particle distance $r=\alpha$ and angle $\theta=\theta_{\alpha}, \theta_{\alpha}+\pi\left(\theta_{\alpha}=0\right.$ in this case $)$;

2. For $\alpha \neq 0$ there are special inter-particle distances, $r$ (besides the zero at the origin) where all pair distribution functions cross, irrespective of their angle $\theta$ dependence.

3. Extremely interesting is the behavior of the angleaveraged pair distribution function $\bar{g}(r)$ as a function of $\alpha$. One notes that the major peak of $\bar{g}(r)$ simply shifts to larger distances (without any sizeable change in its height) as $\alpha$ is increased. For smaller distances $\bar{g}(r)$ starts to develop a shoulder 
that is quite visible for $\alpha=3$ contrary to what seen in Ref. 8 for a slightly larger $\alpha=3.2$, where the shoulder should had been even larger.

4. For small- $r, g(\mathbf{r})$ has almost no angular dependence, and for $\alpha \neq 0, g(r \approx 0, \theta) \simeq C_{\alpha} r^{2}$ for $0 \leq r \leq 0.5$, where $C_{\alpha} \simeq 0.026 \alpha^{2.5}$; when $\alpha=0$, $g(r \approx 0) \propto r^{6}$ as expected. These results derive immediately from the 1-fold vanishing of the BRS wave function when two electrons come close, as opposed to the 3-fold vanishing of Laughlin's wave function, since for small distances only two-body correlations are important. The absence of angular dependence on $g(r, \theta)$ for small $r$ can be easily understood by noting that in the small- $r$ limit: $g(r \approx 0, \theta) \propto \exp \left[u_{s}(r \approx 0, \theta)\right]$, where $u_{s}(r, \theta)$ is given from Eq. (18) and does not have any angular dependence. By recalling that $\lim _{r \rightarrow 0} K_{0}(Q r)=$ $-\ln (Q r / 2)-\gamma$, where $\gamma=0.5772 \ldots$ is the Euler's constant one can easily understand why $g(r, \theta)$ has a quadratic dependence on $r$ and not any angular dependence for small $r$ and values of $\alpha \neq 0$. Although such quadratic dependence at short $r$ is also characteristic for a Wigner crystal state at such filling factor 12 we note that the BRS state does not represent a crystalline state and the overall pair distribution function of the BRS state is strikingly different from the pair distribution function of the Wigner crystal state.

In Fig. 2 we plot the static structure factor $S(\mathbf{q})$ for $\alpha=2$ (top, center panels), and the angle-averaged static structure factor $\bar{S}(q)$ corresponding to $\alpha=0,1,2$ and 3 (bottom panel). The most important feature is the emergence of peaks in $S(\mathbf{q})$ characteristic of a nematic structure. Broadly speaking, the major peak of the $\bar{S}(q)$ shifts to smaller $q$ and its height raises when $\alpha$ is increased, with no significant change in the small- $q$ behavior.

\section{B. Energy of BRS states}

One can compute the correlation energy per particle either directly from Eqs. (3), (5), or (8) to determine the energy per electron for arbitrary values of the BRS parameter $\alpha$, the 2D system width $\lambda$, and Landau level index $L$. The following simplified formula can be used in view of Eq. (7):

$$
E_{\alpha}^{L}(\lambda)=\frac{1}{4 \pi} \int_{0}^{\infty} d q q \tilde{V}(q, \lambda)\left[L_{L}\left(\frac{q^{2}}{2}\right)\right]^{2}[\bar{S}(q)-1]
$$

where $\tilde{V}(q, \lambda)=\left(2 \pi e^{2} / \epsilon q\right) \exp (-\lambda q)$ is the 2D FT of the ZDS interaction potential.18 In addition to allowing straightforward calculations to be extended to any LL, Eq. (23) permits higher numerical accuracy on the calculation of $E_{\alpha}$ since $\bar{S}(q)$ saturates exponentially to 1 for relatively small values of $q$ as compared to $\bar{g}(r)$.

Figure 3 shows the energy difference between BRS states with $\alpha=1,2$ and 3 , and the isotropic state with $\alpha=0$. Our findings indicate that in the $\operatorname{LLL}(L=0)$ the Laughlin state is stable for any $\lambda$, since all $\alpha \neq 0$ states have higher energies (top panel), contrary to prior results that the BRS state for $\alpha=1$ has a lower energy than the Laughlin state if one considers the ZDS potential, with $\lambda>\lambda_{c}=4.1 \pm 1.5$.

The situation changes drammatically in higher LLs $(L \geq 1)$. For small $\lambda$ BRS states have lower energies and the incompressible Laughlin-like state is unstable (see lower panels of Fig. 3). The presence of this instability towards a BRS state may be related to the absence of FQHE states in higher LLs, since for $\alpha \neq 0$ highly damped How-energy modes exist in the resulting nematic system. 1 It is worth noting that for $\lambda \lesssim 1$ the highest investigated $\alpha$ has the lowest energy. In this regime, we are therefore unable to determine the optimal state (even within this familty of trial wavefunctions). This BRS instability may be indicative of a transition towards a completely different state (e.g. as in Ref. 17).

At this point it is important to comment on how precise our determination of these energy differences is. The reader should note that the $\mathrm{HNC} / 0$ approximation is essentially a variational method that always gives an energy that constitutes an upper bound to the exact ground state energy. 11 For example: for the Laughlin state with $\nu=1 / 3$ and $\lambda=0, \mathrm{HNC} / 0$ gives an adimensional correlation energy of -0.405 , while the "exact" value (determined by Monte Carld21) is -0.410. Similar errors (ca. $1 \%$ ) will be present for $\alpha \neq 0$ as well. While an error of this magnitude seems to be of the same order as, or bigger than, the energy differences we are interested in, we remark that these are not uncorrelated errors but systematic deviations due to the nature of the approximations used, and energy differences are considerably more precise. Preliminary results using Monte Carlo simulations 22 for a handful of cases indicate that energy differences are, indeed, significant.

\section{CONCLUSIONS, EXTENSIONS AND FURTHER DEVELOPMENTS}

In conclusion, we applied the HNC theory to study possible BRS states in a 1/3-filled LL. We find that the isotropic Laughlin state is stable in the LLL for realistic interaction potentials. In higher LLs, instabilities towards a BRS state are possible. Since BRS states are gapless 10 this may be a simple explanation why no FQHE was observed for 1/3-filled higher LLs. One caveat is that the magnitude of the energy differences obtained is comparable to the absolute accuracy in the determination of individual energies by the HNC method. Although we believe that energy differences are yielded more precisely than the energies themselves, these results need confirmation by alternative (albeit more time-consuming) methods. Monte Carlo simulations with large number of electrons are currently being performed.22

While these results are by themselves compelling, the connection to recent obsevations of liquid cristalline 
phases in half- and quarter-filled LLs requires more sophisticated methods. One possibility is to geperalize MJ's approach to composite fermion (CF) states, 23 e.g.

$$
\begin{aligned}
& \Psi_{\alpha}^{1 / 2}\left(z_{1}, \ldots, z_{N}\right)= \\
& P_{L} \prod_{j<k}\left(z_{j}-z_{k}+\alpha\right)\left(z_{j}-z_{k}-\alpha\right) \operatorname{Det}\left[\phi_{\mathbf{k}}\left(\mathbf{r}_{i}\right)\right]_{k<k_{F}}
\end{aligned}
$$

where $\phi_{\mathbf{k}}\left(\mathbf{r}_{i}\right)$ are plane waves of CFs, filled for $k<k_{F}=$ $(4 \pi \rho)^{1 / 2}$, and $P_{L}$ projects onto the $L^{\text {th }}$ LL. This wavefunction is an obvious starting point to study the nematic quantum Hall liquid crystals at half filling. For the RIQHE observed near 1/4 filling, similar generalizations are possible. The presence of the Slater determinant in the trial (in addition to the Jastrow factors) implies the need to use the considerably more complex Fermi HNC11,24. Falculations are under way for trial states of these form.25

\section{ACKNOWLEDGMENTS}

We would like to acknowledge helpful discussions with A.T. Dorsey, M. Fogler and L. Radzihovsky. This work was supported by the University of Missouri Research Board.

${ }^{1}$ M.P. Lilly, K.B. Cooper, J.P. Eisenstein, L.N. Pfeiffer, and K.W. West, Phys. Rev. Lett. 82, 394 (1999).

${ }^{2}$ R.R. Du, D.C. Tsui, H.L. Stormer, L.N. Pfeiffer, K.W. Baldwin, and K.W. West, Solid State Comm. 109, 389 (1999).

${ }^{3}$ M. Shayegan, H.C. Manoharan, S.J. Papadakis, E.P. DePoortere, Physica E 6, 40 (2000).

${ }^{4}$ J.P. Eisenstein, M.P. Lilly, K.B. Cooper, L.N. Pfeiffer and K.W. West, Physica A 9, 1 (2001).

${ }^{5}$ E. Fradkin and S.A. Kivelson, Phys. Rev. B 59, 8065 (1999).

${ }^{6}$ K.B. Cooper, M.P. Lilly, J.P. Eisenstein, L.N. Pfeiffer and K.W. West, Phys. Rev. B 60, 11285 (1999).

${ }^{7}$ C. Wexler and A.T. Dorsey, Phys. Rev. B, Sep/15/2001 (in press), preprint: cond-mat/0009096.

${ }^{8}$ K. Musaelian and R. Joynt, J. Phys.: Condens. Matter 8, L105 (1996).

${ }^{9}$ R.B. Laughlin, Phys. Rev. Lett. 50, 1395 (1983).

${ }^{10}$ M.M. Fogler, V.M. Vinokur, Phys. Rev. Lett. 84, 5828 (2000); M.M. Fogler, preprint cond-mat/0107306 (unpublished).

${ }^{11}$ G. Ripka, Physics Reports 56, 1 (1979).

${ }^{12}$ R.B. Laughlin, in The Quantum Hall Effect, ed. by R.E. Prange and S.M. Girvin (Springer-Verlag, 1987), p. 233.

13 J.M. Caillol, D. Levesque, J.J. Weis, and J.P. Hansen, J. Stat. Phys. 28, 325 (1982).

14 J.P. Hansen and D. Levesque, J. Phys. C 14, 603 (1982).

15 J.G. Zabolitzky, Adv. Nucl. Phys. 12, 1 (1981).

16 T. Chakraborty, Phys. Rev. B 31, 4026 (1985).

17 M.M. Fogler, and A.A. Koulakov, Phys. Rev. B 55, 9326 (1997).

18 F.C. Zhang and S. Das Sarma, Phys. Rev. B 33, 2903 (1986).
${ }^{19}$ We use the standard convention for the 2D FT: $\tilde{f}(\mathbf{q})=$ $\int d^{2} r \exp [-i \mathbf{q} \cdot \mathbf{r}] f(\mathbf{r}), f(\mathbf{r})=\int d^{2} q /(2 \pi)^{2} \exp [i \mathbf{q} \cdot \mathbf{r}] \tilde{f}(\mathbf{q})$.

20 For half-filled LLs, more appropriate trial wavefumctions may start with a composite fermion type state 23 with anisotropic Jastrow factors [similar to Eq. (1)] and an isotropic Slater determinant. These type of wavefunctions require use of the considerably more complex Fermi HNC, 1124 see comments in Sec. V.

${ }^{21}$ D. Levesque, J.J. Weis, and A.H. MacDonald, Phys. Rev. B 30, 1056 (1984).

${ }^{22}$ A.J. Schmidt, O. Ciftja, and C. Wexler, in preparation.

${ }^{23}$ Composite Fermions, ed. O. Heinonen (World Scientific, New York, 1998).

${ }^{24}$ O. Ciftja, and S. Fantoni, Phys. Rev. B, 58, 7898 (1998).

${ }^{25}$ O. Ciftja, and C. Wexler, in preparation. 


\section{FIGURES}

(a)

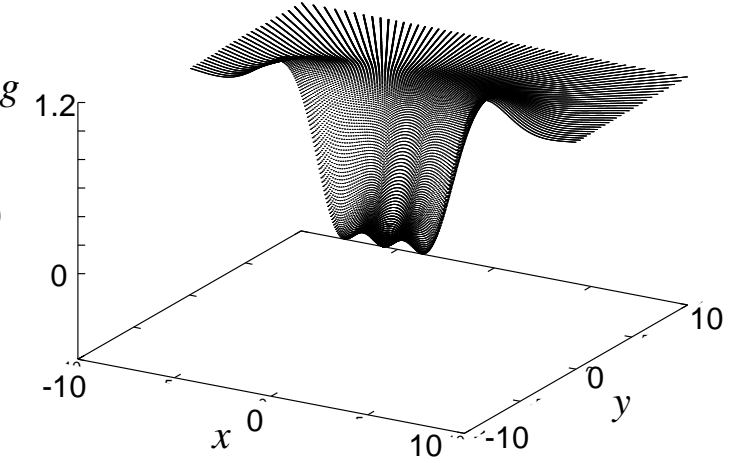

(b)

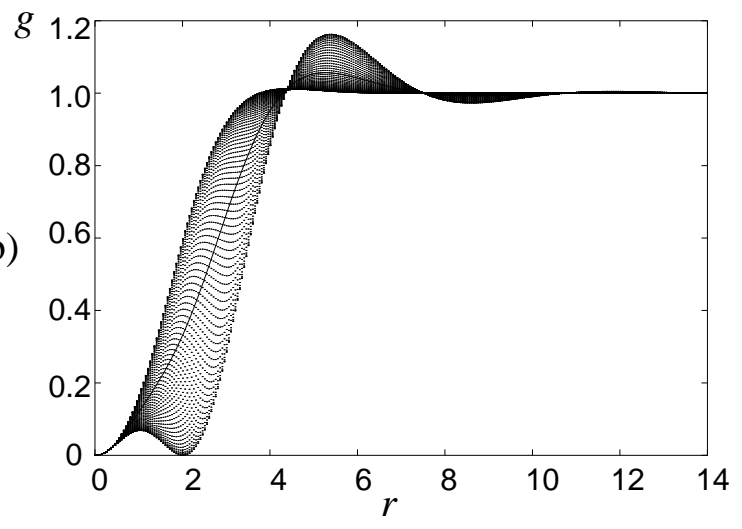

(c)

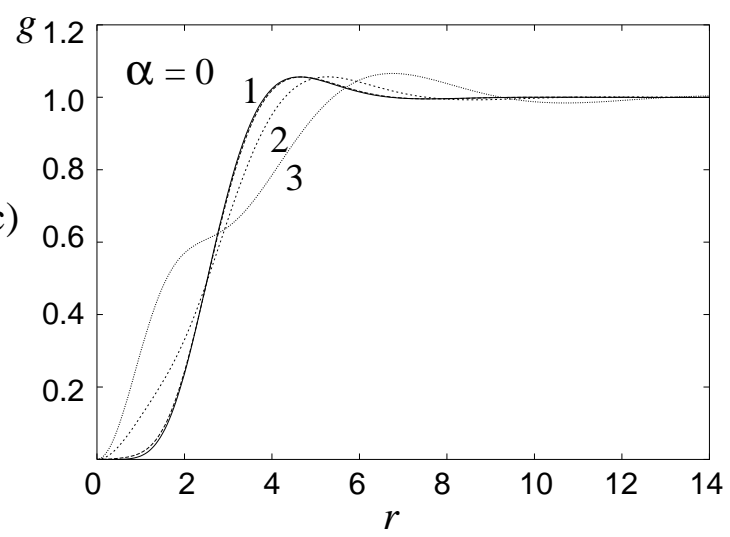

FIG. 1. Pair distribution function for the BRS state at $\nu=1 / 3$. (a) $\alpha=2$, surface plot of $g(r, \theta)$ (the surface for $y<0$ was removed for clarity); (b) $\alpha=2$, dotted lines: $g(r, \theta)$ for various $\theta \in[0,2 \pi]$, full line: angle averaged $\bar{g}(r)$; (c) Angle averaged $\bar{g}(r)$ for $\alpha=0,1,2$ and 3 ( 0 and 1 are virtually identical). Note the discrete nodes of $g(r, \theta)$ at $r=\alpha$, $\theta=\theta_{\alpha}, \theta_{\alpha}+\pi\left(\theta_{\alpha}=0\right.$ in this case). Calculations were performed in the $\mathrm{HNC} / 0$ approximation. (a)
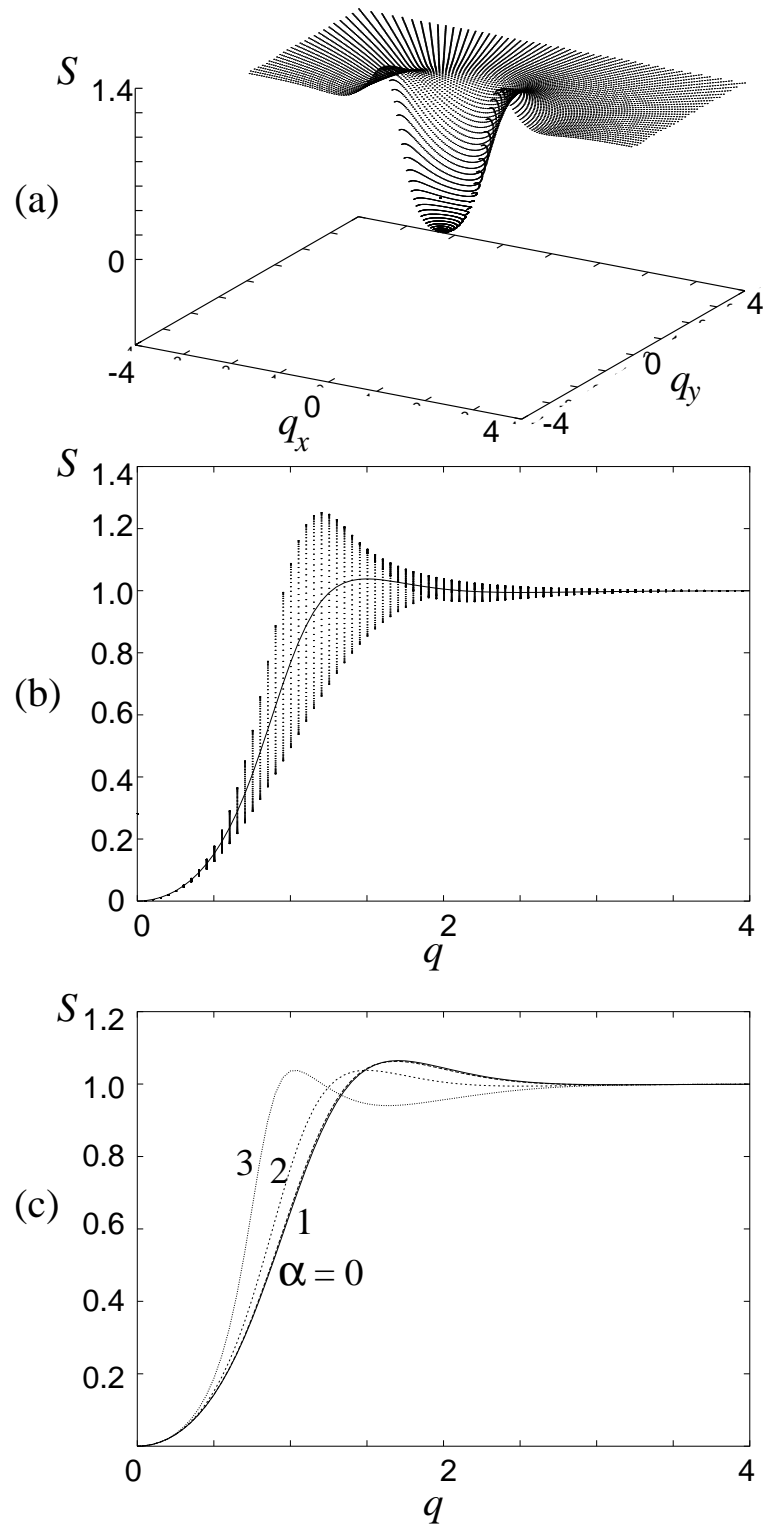

FIG. 2. Static structure factor for the BRS state at $\nu=1 / 3$. (a) $\alpha=2$, surface plot of $S(r, \theta)$ (the surface for $q_{y}<0$ was removed for clarity); (b) $\alpha=2$, dotted lines: $S\left(q, \theta_{q}\right)$ for various $\theta_{q} \in[0,2 \pi]$, full line: angle averaged $\bar{S}(q)$; (c) Angle averaged $\bar{S}(q)$ for $\alpha=0,1,2$ and 3 (0 and 1 are virtually identical). Note the presence of peaks in $S(\mathbf{q})$ consistent with a nematic structure. Calculations were performed in the $\mathrm{HNC} / 0$ approximation. 

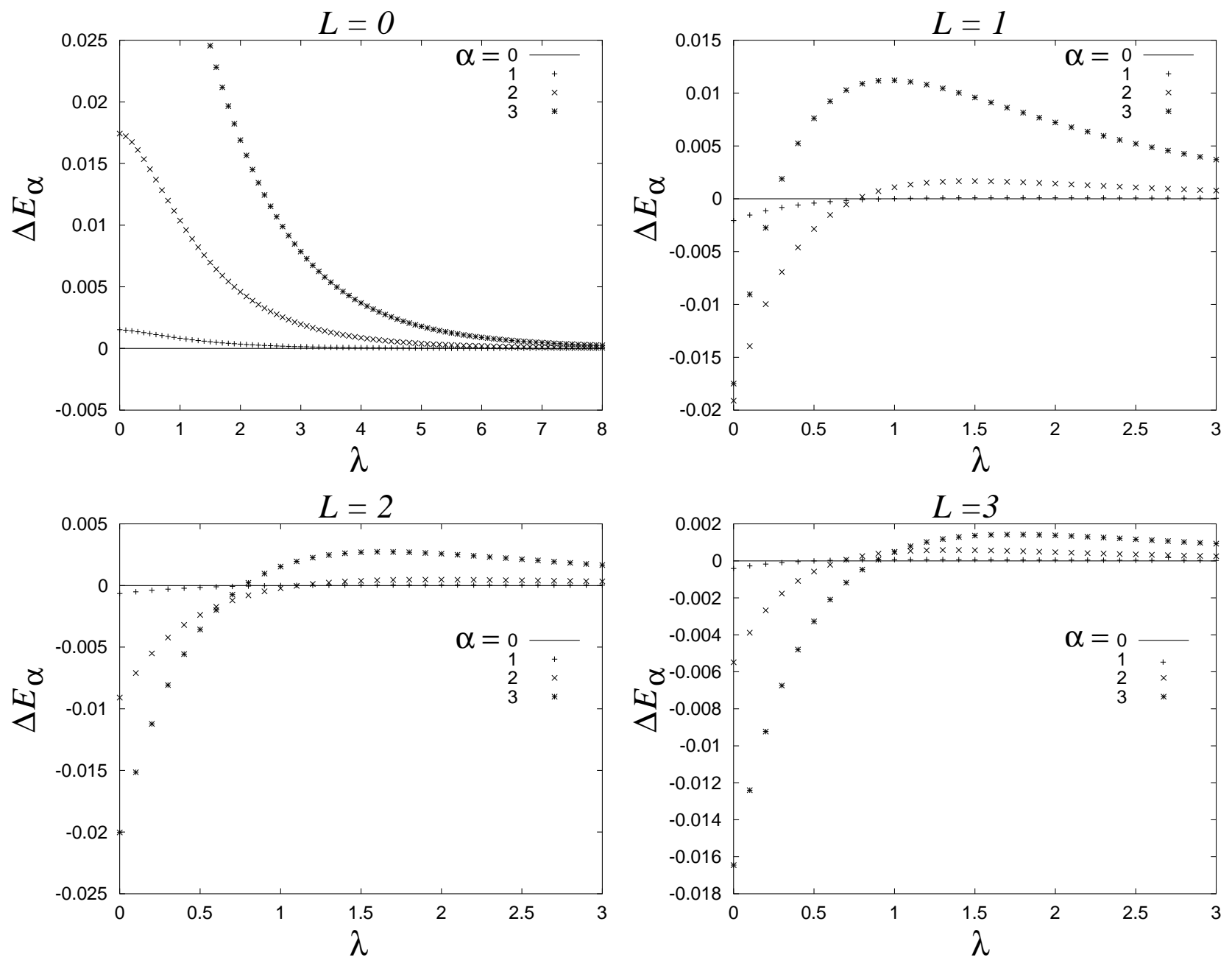

FIG. 3. Energy per particle in BRS states with $\alpha=1,2$ and 3 relative to the isotropic $(\alpha=0)$ state: $\Delta E_{\alpha}(\lambda)=E_{\alpha}(\lambda)-E_{0}(\lambda)$ for various Landau levels $L$ as functions of the short distance cut-off $\lambda$ [Eq. (23)]. Energies are in units of $e^{2} /\left(\epsilon l_{o}\right)$. Note that in the LLL $(L=0)$ BRS states are always higher in energy, whereas in higher LLs $(L \geq 1)$ there are ranges of $\lambda$ for which BRS states are favorable. 